\title{
EFFECT OF HYDROSTATIC PRESSURE ON HALOTHANE-INDUCED DEPRESSION OF MITOCHONDRIAL RESPIRATION
}

\author{
Peter J. Cohen \\ Department of Anesthesiology \\ University of Michigan Medical School \\ Ann Arbor, Michigan 48109 \\ (Received in final form December 28, 1982)

\section{Summary}

\begin{abstract}
Anesthetized animals are awakened when subjected to increased atmospheric pressure. Whether all phenomena associated with the anesthetic state are similarly reversed is not known. Since the anesthetic halothane produces a dose-related reversible depression of rat liver mitochondrial respiration, the effect of 51 atmospheres of pressure on the drug's action was evaluated. It is concluded that application of pressure does not antagonize the inhibition produced by this anesthetic.
\end{abstract}

The ability of high pressure to antagonize anesthetic action (pressure reversal) is well documented. Restoration of the light output of anesthetized luminous bacteria by application of 2000-4000 atmospheres (ATA) (1), pressure-induced recovery of swimming in tadpoles treated with 2.5 percent ethanol (200-300 ATA) (2) or 0.5-1.5 percent halothane (35-171 ATA) (3), reversal of halothane's inhibition of axonal transmission (3569 ATA) (4), and decreased potency of nitrous oxide (5) and isoflurane (6) in the mouse (25-100 ATA) are but a few examples. However, pressure reversal is not universal. Neither halothane's inhibition of synaptic transmission in the rat superior cervical ganglion (4) nor its depression of ciliary beat in Tetrahymena pyriformis (7) is restored by pressures of 35-137 ATA. Thus, different effects of anesthetics (and pressure) may reflect actions at multiple sites (8). Volatile anesthetics reversibly inhibit state 3 mitochondrial respiration. In vitro (ED-50) and in vivo (the alveolar concentration required to abolish the response to noxious stimuli) potencies are similar; both are related directly to lipid solubility (9). Since not all anesthetic action appears amenable to pressure reversal, and since the interaction of anesthesia and pressure on mitochondrial respiration has not been described, the following study was performed.

\section{Methods}

State 3 mitochondrial respiration $(Q)$ was determined in rat liver mitochondria exposed to air $(n=9)$ and in mitochondria equilibrated with 0.9 percent halothane (2-bromo-2chloro-1,1,1-trifluoroethane) vaporized in air $(n=9)$ as previously described (9). In each study, Q was measured at ambient pressure (QA), after compression to 51 ATA (QP1), and following decompression (QP2). Evaluation of QA and QP2 was accomplished by direct measurement. Since it was not possible to analyze mitochondrial oxygen tension in samples under pressure, the value of $\mathrm{QPl}$ was obtained by indirect means as described below.

Respiring mitochondria were compressed after being drawn into a syringe which was put in a water-filled steel chamber capable of hydraulic compression (3). Because it was the liquid phase which was exposed to pressure, significant changes in the partial pressures of oxygen, nitrogen, or halothane did not occur. In order to avoid the alterations of mitochondrial function which follow exposure to in vivo temperature, a constant 
temperature of $5 \mathrm{C}$ was provided by placing the chamber in a thermostatically controlled water bath. Immediately prior to compression, $0.5 \mathrm{ml}$ of the mitochondrial suspension in a $1 \mathrm{ml}$ syringe was mixed anaerobically with $4.5 \mathrm{ml}$ of reaction medium (9) in a $5 \mathrm{ml}$ glass syringe and state 3 respiration thereby initiated. Care was taken to prevent bubble formation. Substrate (glutamate), inorganic phosphate, oxygen and adenosine diphosphate were present in excess. The aliquot which was to be hydrostatically compressed (sample $P$ ) was than transferred anaerobically to a $2 \mathrm{ml}$ glass syringe which was capped and placed in the pressure chamber. The syringe with the remaining suspension (sample A) was capped and put in the water bath. The chamber was closed but not pressurized. An aliquot of sample $A$ was introduced into a thermostatically jacketed (perfused from the water bath) oxygen electrode (Radiometer E5046) for the measurement of mitochondrial respiration under ambient conditions (QA). The chamber was pressurized within 10 seconds (time $=0$ ), and measurements of the oxygen tension of sample $A$ recorded every 30-60 seconds. Analysis of sample A was continued during the 7 minute period of compression, a duration which was identical for anesthetized and nonanesthetized mitochondria. Decompression was accomplished within 10 seconds, the syringe removed, and timed measurements of the oxygen tension of sample P made.

The rate of change of oxygen tension of sample A (evaluated by by regression analysis) was used to calculate $\mathrm{QA}$ (figure 1). The rate of change of oxygen tension of sample $\mathrm{P}$ following decompression, determined by regression analysis $(r=0.97-1.00)$, was used to calculate QP2 and to estimate the oxygen tension of sample $P$ at the moment of decompression. In a similar fashion, regression analysis of the data from sample $A$ was used to calculate the oxygen tension of sample $\mathrm{P}$ at the time of compression (the oxygen tension being the same in samples $A$ and $P$ ). QPI was determined indirectly using the difference in oxygen tensions of sample $\mathrm{P}$ at the times of compression and decompression and the duration of compression (figure 1).

\section{Calculation of mitochondrial oxygen uptake}

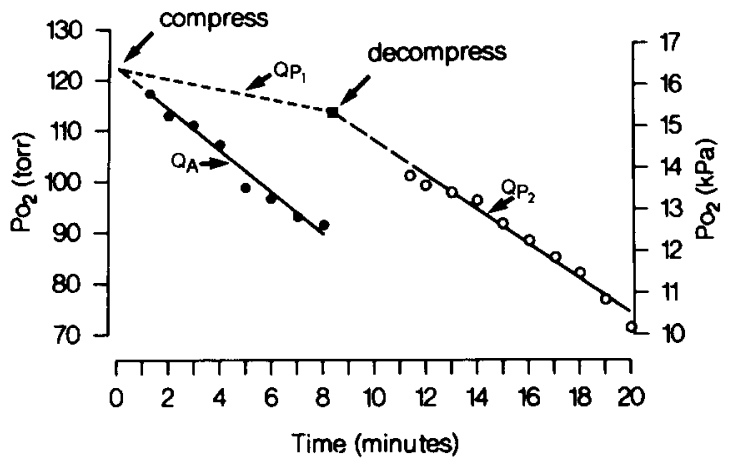

FIG. 1

Analysis of state 3 mitochondrial oxygen uptake using data from mitochondria at ambient pressure (๑) and following decompression from 150 ATA (O). QA and QP2 are determined using the measured rates of change of oxygen tension (solid lines). The oxygen tension of samples $\mathrm{A}$ and $\mathrm{P}$ at the time of compression (which are identical) are calculated using the data from sample A. Oxygen tension at the instant of decompression $(\boldsymbol{G})$ is evaluated by extrapolating the values of sample P2 (solid line) to the point of decompression (bold hatched line). QP1 is evaluated using the duration of compression and the oxygen tension of sample $\mathrm{P}$ at the times of compression and decompression. The rate of mitochondrial respiration is decreased during compression to 150 ATA and does not return completely to normal following decompression. These data were obtained in a single study; each point represents the oxygen tension at a single point of time. 


\section{$\underline{\text { Results }}$}

Neither compression to 51 ATA nor decompression resulted in altered state 3 respiration in the anesthetized and non-anesthetized suspensions (Table I). The inhibition of mitochondrial respiration produced by halothane in these experiments was similar to that reported previously (9), and persisted both during and after compression.

\section{TABLE I}

Effect of 51 ATA and Halothane on Mitochondrial Respiration

\begin{tabular}{|c|c|c|c|c|}
\hline $\begin{array}{l}\text { QA } \\
\text { QP1 } \\
\text { QP2 }\end{array}$ & $\begin{array}{l}\text { CONTROL } \\
1.53 \pm 0.08^{* *} \\
1.26 \pm 0.11 \\
1.48 \pm 0.12\end{array}$ & $\begin{array}{c}\text { HALOTHANE } \\
1.04+0.10 \\
0.93 \pm 0.08 \\
0.86 \pm 0.09\end{array}$ & $\begin{array}{l}\text { CHANGE } \\
-0.49 \pm 0.13 \\
-0.33 \pm 0.14 \\
-0.62 \pm 0.15\end{array}$ & $\begin{array}{l}\mathrm{P}^{*} \\
<.005 \\
<.05 \\
<.001\end{array}$ \\
\hline $\begin{array}{l}\mathrm{QP1}-\mathrm{QA} \\
\mathrm{QP} 2 \text { - } \mathrm{QA} \\
\mathrm{QP} 2 \text { - } \mathrm{QP} 1\end{array}$ & $\begin{array}{r}-0.27 \pm 0.14 \\
-0.04 \pm 0.08 \\
0.22 \pm 0.18\end{array}$ & $\begin{array}{l}-0.11+0.07 \\
-0.18 \pm 0.10 \\
-0.07 \pm 0.11\end{array}$ & & \\
\hline $\begin{array}{l}\mathrm{F}^{* * *} \\
\mathrm{P}^{* * *}\end{array}$ & $\begin{array}{l}1.821 .02 \\
>.1>.25\end{array}$ & & & \\
\hline
\end{tabular}

Although 51 ATA alone did not significantly affect mitochondrial respiration either during compression or following decompression, higher pressures had a pronounced action (figure 1). Compression to 150 ATA decreased $\mathrm{Q}$ by an average of 62 percent. Following decompression from 150 ATA, mean oxygen uptake remained 21 percent below control ( $\mathrm{p}$ $<0.005$ when QA, QP1 and QP2 are compared by analysis of variance). Pressure, therefore, may have an action on mitochondrial respiration separate from that of halothane, a phenomenon which must be considered when analyzing these data.

\section{Discussion}

Prior to compression, the two samples ( $A$ and $P$ ) had been treated identically. Oxygen tension of the reaction medium alone was unaltered following a 7 minute period of compression. Thus, pressure itself would not have been expected to affect oxygen tension; any observed changes would relate to alterations in the rate of mitochondrial respiration. Since compression was carried out in the liquid phase, and since the volume of mitochondrial suspension was considerably less than the total volume of water in the thermostatically controlled bath, temperature changes in the respiring suspension would not be anticipated. The oxygen electrode was stable (varying less than 2 torr during a 30 min period), had an appropriate response time (time constant for a change from $0-150$ torr $=47$ seconds) and did not appear sensitive to halothane (10). Assuming that the effects of compression and decompression are immediate, changes in the rate of decrease of oxygen tension during the time of compression should reflect alterations of $\mathrm{Q}$ produced by halothane and/or pressure.

There is little evidence that depression of mitochondrial respiration is directly responsible for the anesthetic state. However, the significant correlation between in vivo and in vitro anesthetic potency, the similar relationship of in vivo and in vitro potency to lipid solubility (9), and the observation that lipophilic compounds which do not influence mitochondrial respiration are devoid of in vivo anesthetic action (11) make it likely that both in vivo and in vitro anesthetic action share. a common mechanism. The data presented in this study provide an added complexity to this hypothesis since they suggest that reversal of anesthetic-induced inhibition of mitochondrial respiration and the awakening of an anesthetized animal are not parallel effects of hydrostatic pressure. 
One ought not extrapolate from these data to a general since only the interaction of one pressure and one concentration of halothane was evaluated. It is possible that exposure to a higher pressure (or lower halothane concentration) might have allowed demonstration of pressure reversal. However, although a pressure of 51 ATA was without effect on $\mathrm{Q}$, more pronounced compression is not benign. Disruption of mitochondrial membranes with release of endogenous enzymes is produced by hydraulic compresion to 483-966 ATA (12). A significant and only partially reversible decrease in $Q$ was observed at 150 (but not 51) ATA in this study, perhaps related to the aforementioned physical and biochemial changes. Thus, the apparent absence of pressure antagonism might possibly represent an occult depression of Q by 51 ATA interacting with a small degree of pressure reversal. Whether this explains the apparent failure of pressure to antagonize halothane's effect cannot be resolved at the present time. Perhaps exposure to lower pressures (e.g. 100 ATA), and for a different duration, might have produced pressure reversal. However, compression to 51 ATA has been demonstrated to antagonize in vivo and in vitro anesthetic action, and therefore the in vitro effect of this magnitude of pressure seemed relevant.

In summary, it is concluded that under the experimental conditions of this study, application of a hydrostatic pressure of 51 ATA did not reverse the inhibition of mitochondrial respiration produced by 0.9 percent halothane.

\section{Acknowledgment}

This work was performed during a Leave of Absence spent in the Division of Anaesthesia, Clinical Research Centre, Harrow, Middlesex HA1 3UJ, United Kingdom. The considerable encouragement and assistance of Doctors John F. Nunn and Michael J. Halsey is gratefully acknowledged.

\section{References}

1. F.H. JOHNSON, D.E.S. BROWN and D.A. MARSLAND, J. Cell. Comp. Physiol. 20 269-276 (1942).

2. F.H. JOHNSON and E.A. FLAGLER, Science 112 91-92 (1950).

3. M.J. HALSEY and B. WARDLEY-SMITH, Nature $257811-813$ (1975).

4. J.J. KENDIG, and E.N. COHEN, Molecular Mechanisms of Anesthesia, edited by B.R. FINK, pp. 421-427, Raven Press, N.Y. (1975).

5. M.J. HALSEY, E.I. EGER, D.W. KENT and P.J. WARNE, Molecular Mechanisms of Anesthesia, edited by B.R. FINK, pp. 353-361, Raven Press, N.Y. (1975).

6. D.W. KENT, M. J. HALSEY, E.I. EGER and B. KENT, Anesth. Analg. Curr. Res. 56 97-101 (1977).

7. W.D.B. POPE, A.J. JONES, M.J. HALSEY and J.F. NUNN, Canad. Anaesth. Soc. J. $25319-322(1978)$.

8. M.J. HALSEY, B. WARDLEY-SMITH and C.J. GREEN, Brit. J. Anaesth. 50 10911097 (1978).

9. M.L. NAHRWOLD and P.J. COHEN, Anesthesiology 38 437-444 (1973).

10. R.D. MCHUGH, R.M. EPSTEIN and D.E. LONGNECKER, Anesthesiology 50 47-49 (1979).

11. M.L. NAHRWOLD, J.A. Rapiejko and P.J. COHEN, Molecular Mechanisms of Anesthesia, edited by B.R. FINK, pp. 431-438, Raven Press, N.Y. (1975).

12. M. BRONFMAN and H. BEAUFAY, FEBS Letters 36 163-168 (1973). 